\title{
Análisis del estado del turismo para la construcción de estrategias sostenibles Vaupés, Colombia
}

\author{
Analysis of the state of tourism for the construction of sustainable \\ strategies Vaupés, Colombia
}

\author{
Análise do estado do turismo para a construção de estratégias \\ sustentáveis Vaupés, Colombia
}

Hernando Castro-Garzón ${ }^{1}$; Francy L. Montealegre-Torres ${ }^{2}$

1 Administrados de Empresas Agroindustriales, Esp, MSc, (c) PhD, Profesor Ocasional de la Universidad de los Llanos, de la Facultad de Ciencias Económicas en el programa de Administración de Empresas. Villavicencio, Colombia.

2 Administrador de Empresas, Msc(e), en Gestión de Desarrollo Sustentable, Universidad Autónoma de Guerrero. Acapulco, México.

Email: hcastro@unillanos.edu.co

Recibido: 02 de agosto de 2018

Aceptado: 07 de diciembre de 2018

\begin{abstract}
Resumen
Se busca identificar en el departamento del Vaupés las diferentes oportunidades, amenazas, fortalezas y debilidades -en cuanto a turismo- por medio de un diagnóstico en donde se realiza una revisión abordando lo político, cultural, ambiental, geográfico e histórico para finalizar con un modelo estratégico adaptado a seis etapas a desarrollar; en la primera etapa se observa la ubicación geográfica y se han señalado las zonas con actividad intensiva, extensiva y primitiva, durante la segunda etapa se realiza un plan de los posibles sitios a visitar demarcándolos en el mapa correspondiente, la tercera fase hace énfasis a la arquitectura que debería de usarse en el lugar para el desarrollo del turismo; dicho esto la cuarta etapa establece los mecanismos para la generación de ingresos seguido por el manejo y monitoreo del impacto de los visitantes y finaliza con los aspectos que deben tener los guías naturalistas. Así las cosas, se hizo uso de una metodología descriptiva y exploratoria para tener un mayor alcance; se empleó el manual de desarrollo del ecoturismo creado por "The Nature Conservancy"; además de aprovechar la técnica etnocultural como base de la infraestructura apoyados en procesos computarizados para su diseño.
\end{abstract}

Palabras clave: turismo, Etnoturismo, turismo gastronómico, DOFA, estrategias, plan turístico.

\begin{abstract}
It is sought to identify in the department of Vaupés the different opportunities, threats, strengths and weaknesses -in terms of tourism- by means of a diagnosis where a review is made addressing the political, cultural, environmental, geographical and historical to finalize with a model strategic adapted to six stages to develop; In the first stage the geographical location
\end{abstract}


is observed and the areas with intensive, extensive and primitive activity have been indicated. During the second stage, a plan of the possible sites to be visited is demarcated in the corresponding map, the third phase makes emphasis on the architecture that should be used in the place for the development of tourism; said this the fourth stage establishes the mechanisms for the generation of income followed by the management and monitoring of the impact of visitors and ends with the aspects that naturalist guides should have. Thus, a descriptive and exploratory methodology was used to have a greater scope; the ecotourism development manual created by "The Nature Conservancy" was used; besides taking advantage of the ethnocultural technique as a base of the infrastructure supported by computerized processes for its design.

Key words: Tourism, ethno, gastronomic tourism, FODA, strategies, tourist plan.

\section{Resumo}

Busca-se identificar no departamento de Vaupés as diferentes oportunidades, ameaças, pontos fortes e fracos - em termos de turismo - por meio de um diagnóstico onde se faz uma revisão abordando o político, cultural, ambiental, geográfico e histórico para finalizar com um modelo estratégico adaptado a seis etapas para desenvolver; No primeiro estágio a localização geográfica é observada e as áreas com atividades intensivas, extensas e primitivas têm sido indicadas.Na segunda etapa, um plano dos possíveis locais a serem visitados é demarcado no mapa correspondente, a terceira fase faz ênfase na arquitetura que deve ser usada no lugar para o desenvolvimento do turismo; A quarta etapa estabelece os mecanismos para a geração de renda, seguida pela gestão e monitoramento do impacto dos visitantes e termina com os aspectos que os guias naturalistas devem ter. Assim, uma metodologia descritiva e exploratória foi utilizada para ter um escopo maior; o manual de desenvolvimento de ecoturismo criado por "The Nature Conservancy" foi usado; além de aproveitar a técnica etnocultural como base da infraestrutura suportada por processos informatizados para o seu design.

Palavras chave: Turismo, etnoturismo, turismo gastronômico, DOFA, estratégias, plano de turismo.

\section{Introduccion}

La construcción de sostenibilidad en el turismo no ha sido llevada a feliz término en el ámbito latinoamericano (Luck y Kirstages, 2002); ya que como es constante en esta región los proyectos creados no han sido finalizados. Uno de los factores es la no inclusión de la capacidad de carga como indicador a tener en cuenta, "El turismo sostenible ha revitalizado el concepto de capacidad de carga, aunque todavía existen muchas dudas respecto a sus aplicaciones potenciales" (López y López, 2008). Por otro lado la ausencia de legislación en el ámbito turístico para las zonas de protección crea vacíos para la ejecución de dichos proyectos (Nello, 2003), con el agravante que los réditos obtenidos por la implementación de este tipo de proyectos afecta a las poblaciones locales. (Narvaez y Sepulveda, 2002).

En adicion a lo anterior, se entiende como turismo sostenible al cuidado y aprovechamiento de los recursos, "un turismo que mantiene un equilibrio entre los intereses sociales, económicos y ecológicos, integrando las actividades económicas y recreativas con el objeto de buscar la conservación de los valores naturales y culturales" (AIEST,1991) siendo ésta la mejor forma de generar apalancamiento para las regiones más apartadas; reafirmándolo en la OMT (1998) "Para facilitar al visitante una experiencia de alta calidad y mantener la calidad del medio ambiente, del que tanto la comunidad anfitriona como los visitantes dependen"; siendo sinérgica las acciones entre agentes y actores del territorio.

A su vez el interés por parte de los entes gubernamentales en apoyar el turismo sostenible no es el esperado "las debilidades del concepto radica en que no se cuenta aún con suficiente respaldo en los círculos políticos y de gobierno" (Bertoni, 2008) lo cual genera una debilidad para el desarrollo de los proyectos; más aún la apropiación del turismo sostenible se convierte en un accionar de voluntad política "la condición de hacer sujeto político al turismo sostenible es a fin de cuentas una manifiesta voluntad política" (Ibíd.); mostrando así la injerencia de la institucionalidad en la implementación de este tipo de programas.

Con respecto al enfoque que se le debe dar a las políticas de turismo sostenible se aprecia que deben ser, aparte de fijadores de cultura, creadores de conceptos "La investigación acerca del turismo sustentable evoca innovaciones más radicales que rara vez son aceptadas por los diseñadores de políticas públicas en los gobiernos" (Chávez, 2013) por tanto las visiones deben ser abiertas y respetuosas de los diferentes contextos.

Así que, se tiene como resultado una herramienta que puede potencializar regiones que han sido olvidadas, ya sea por su ubicación geográfica o su bajo margen político de negociación "La sustentabilidad pasa a ser un concepto central que propone la reevaluación del 
papel del turismo en la sociedad contemporánea" (Tarlombani, 2005) siendo ésta, si es manejada de manera adecuada la posibilidad de que estas zonas obtengan visibilidad y un mejoramiento social.

\section{Materiales y métodos}

Para este trabajo se implementó una metodología de tipo cualitativa con dos alcances: uno descriptivo y otro exploratorio. Se seleccionó el descriptivo porque busca especificar las propiedades, características y perfiles de personas, grupos y comunidades o cualquier otro fenómeno que sea sometido a análisis; y exploratorio ya que este se usa cuando el objetivo es examinar un tema o un problema de investigación poco estudiado, ayuda a familiarizarse con fenómenos desconocidos, obtener más información para una investigación más completa (Hernández, 2010)

La investigación se llevó a cabo en cuatro fases de las cuales para el presente escrito se desarrollarán la tercera y cuarta fase como producto de las anteriores, de la siguiente manera:

\section{Primera fase}

Tabla 1. Diagnóstico ambiental.

Señalar el estado actual del entorno social, económico, empresarial, político y ambiental del departamento.

\section{Descripción de la actividad}

Se realizó la consulta de información en las páginas oficiales de la Alcaldía, Gobernación y Ministerios, planes de gobierno de los últimos años, planes de vida indígena de las diferentes asociaciones y demás páginas web con información del departamento.

Se visitaron las comunidades incluidas en la investigación, el relleno sanitario, los pozos para la investigación de la cría de guaracú, mercado tradicional la maloca, instituciones educativas, el hospital y las entidades gubernamentales.

\section{Segunda Fase}

Tabla 2. Inventario turístico.

Elaboración de un Inventario de los lugares y recursos turísticos con los que cuenta el Departamento del Vaupés.

\section{Descripción de la actividad}

Los recursos y los atractivos del departamento en su mayoría se encuentran ubicados en áreas cercanas a la capital del departamento, por lo cual se escogieron cinco comunidades indígenas que cuentan con potencial turístico y donde actualmente llegan la mayor parte de los turistas.

Se recorrieron los espacios urbanos y comunidades indígenas objeto de estudio con el fin de conocer los recursos y lugares turísticos; su estado, su uso y el tipo de turismo que puede ser aplicado en cada uno de ellos.

Se recopilo información primaria en los lugares que se visitaron e información secundaria de aquellos que por recursos y accesibilidad no pudieron reconocer de primera mano. Se organizó según un manual para el diagnóstico de inventarios turísticos del ministerio de Industria, Comercio y Turismo de Colombia.

\section{Tercera fase}

Tabla 3. Diagnóstico DOFA.

Identificar Potencialidades, Amenazas, Fortalezas y Debilidades del Sistema turístico de este departamento.

\section{Descripción de la actividad}

La matriz DOFA es un método de planeación que permite definir los aspectos internos y externos que favorezcan o inhiban el buen funcionamiento de la empresa, la matriz se desglosa de la siguiente manera: (D) debilidades: se refiere a los aspectos internos que de alguna u otra manera no permiten o que frenan el cumplimiento de los objetivos planteados; $(\mathrm{O})$ oportunidades: se refiere a los acontecimientos o características externas objeto de estudio, que puedan ser utilizadas a favor del gestor para garantizar el crecimiento de su empresa; (F) Fortalezas: son las características internas del negocio o territorio que permitan impulsar al mismo y poder cumplir las metas planteadas y por último (A) Amenazas: son los acontecimientos externos del negocio en la mayoría de las veces incontrolables que pueden afectar su desarrollo (Ponce,2007)

Con esta herramienta de planificación lo que se busca es evaluar los aspectos internos y externos, positivos y negativos del sector turístico del departamento específicamente los relacionados con los comunidades cercanas y la misma capital, que intervienen en el desarrollo social y económico de esta región.

Así las cosas, se indagó acerca de los cuatro aspectos con los pobladores de la zona urbana y rural, con el alcalde, representantes legales de operadores turísticos de la región, delegados de la Corporación para el Desarrollo

Sostenible del Norte y el Oriente Amazónico (CDA), la Corporación SINCHI, integrantes de Centro Agropecuario y de Servicio Ambientales del SENA 


\section{Cuarta fase}

Tabla 4. Estrategias de diagnóstico.

Proponer planes y estrategias inclusivas frente a las problemáticas encontradas en el estudio.

\section{Descripción de la actividad}

Se seleccionó para el desarrollo de propuestas y estrategias que permitieran estructurar y dinamizar el sector turístico del departamento, el segundo volumen del "Manual de Desarrollo del Ecoturismo" de la fundación ambiental "The Nature Conservancy"

El manual cuenta con seis grandes temas, se adaptó cada uno de ellos a modo de propuesta a la realidad del departamento, con el fin de servir como guía para la implementación de acciones fundamentales para el desarrollo del sector.

\section{Resultados}

\section{Matriz DOFA}

Los factores encontrados son los siguientes (Tabla 5):

\section{Aspectos Externos}

\section{Oportunidades}

\section{Nuevas tendencias en la demanda}

El viajero de hoy está dejando de frecuentar el turismo convencional, un turismo de playa y zonas urbanas por ofertas mucho más llamativas y desafiantes, con un creciente interés por el turismo vivencial o turismo basado en experiencias; estos turistas están ávidos de un ejercicio innovador que le brinden contacto con la naturaleza y un cambio temporal de estilo de vida. (Moya, 2006)

El departamento del Vaupés se encuentra en ventaja gracias a que puede ofrecer un producto diferenciado y de calidad donde se encuentran inmersas las comunidades indígenas como actores principales (Cabrera, 2014)

\section{Reconocimiento y ayuda gobierno nacional}

En los últimos años el gobierno nacional le ha dado cada vez más importancia al departamento, pues hasta antes de 1998 era un lugar olvidado; a partir de la toma guerrillera efectuada por las FARC “...para adelantar las conversaciones de paz entre la administración Pastrana y las FARC, esta guerrilla tomó por asalto a Mitú, capital del departamento de Vaupés, en el suroriente colombiano" (Echandía, 2004), una situación desafortunada pero que permitió que se hiciera visible dándole paso a las inversiones para espacios deportivos, educativos, sociales y culturales con el fin de mejorar la calidad de vida de los locales y buscando la conservación del ser y el hacer indígena.

\section{Apertura del indígena hacia el turista}

Gracias a la apertura que ha tenido el departamento al turismo las comunidades han ido progresivamente

Tabla 5. Matriz DOFA del Departamento del Vaupés.

\begin{tabular}{|c|c|}
\hline \multicolumn{2}{|c|}{ Aspectos Externos } \\
\hline Oportunidades & Amenazas \\
\hline $\begin{array}{l}\text { - Nuevas tendencias en la demanda } \\
\text { - Reconocimiento y ayuda gobierno nacional } \\
\text { - Formación de la población. } \\
\text { - Apertura del Indígena al Turista. } \\
\text { - El Ecoturismo y el Etnoturismo, mercado en apertura y } \\
\quad \text { expansión. }\end{array}$ & $\begin{array}{l}\text { - Actividad turística no inclusiva y sin planificación } \\
\text { - Deterioro y pérdida de algunas estructuras turísticas. } \\
\text { - Deficiencias en la coordinación y colaboración público- } \\
\text { privada. }\end{array}$ \\
\hline \multicolumn{2}{|c|}{ Aspectos Internos } \\
\hline Fortalezas & Debilidades \\
\hline $\begin{array}{l}\text { - Riqueza y diversidad natural y cultural. } \\
\text { - Producto diferenciador. } \\
\text { - Nuevas Inversiones en infraestructura. }\end{array}$ & $\begin{array}{l}\text { - Escasa existencia de producto desarrollado. } \\
\text { - Insuficiente penetración en los canales de comercialización } \\
\text { turística. } \\
\text { - Infraestructura para el transporte y altos costos. } \\
\text { - Falta de apoyo gubernamental. } \\
\text { - Organización de las comunidades. }\end{array}$ \\
\hline
\end{tabular}

Elaborada por el autor como resultado del análisis del estado del turismo en Vaupés, Colombia (2017). 
abriendo espacios para que puedan acceder los visitantes donde se benefician ambas partes intercambiando vivencias por recursos para el sustento de los indígenas.

\section{El Ecoturismo y el Etnoturismo, mercado en apertura y expansión}

El Ecoturismo y el Etnoturismo han surgido hace algunos años; donde prima la sostenibilidad (Trejo \& Marcani, 2016) preservación y apreciación del medio tanto natural como cultural que acoge y sensibiliza a los viajeros; como es un tipo de turismo relativamente nuevo, es una alternativa turística no masificada y poco saturada que permite un acceso inmediato al actor o promotor que se debe de abrir camino y posicionarse por medio del fomento de los lugares y actividades a disfrutar por parte de potenciales turistas.

Este es el trabajo que hacen algunos en el departamento, pero hace falta hacer un impulso publicitario más agresivo con el fin de ganar reconocimiento en la esfera turística; ciertamente es una oportunidad sin igual para el departamento puesto que las características geográficas con las que cuenta son únicas. (Jiménez, 2007).

\section{Amenazas}

\section{Actividad turística no inclusiva y sin planificación}

Existe una empresa y una persona natural en el municipio de Mitú que se encuentran ofreciendo servicios turísticos a nacionales que se desplazan principalmente desde Caquetá, San José del Guaviare, Villavicencio y Bogotá guiando en actividades de senderismo y avistamiento de aves sin tener en cuenta a las comunidades allí establecidas, lo mismo que no existe una ganancia significativa para estos.

Así las cosas, es importante el contacto directo del indígena con el turista, ya que la experiencia intercultural daría una gran contribución en conocimiento externo y de difusión de las actividades allí realizadas en un marketing voz a voz.

\section{Deterioro y/o pérdida de estructuras turísticas}

La maloca Ipanoré ubicada en la zona urbana de Mitú fuera en su momento la más grande de Latinoamérica; se consumió en un voraz incendio en el mes de abril del año 2016. Allí cada dos años en el mes de octubre se reunían comunidades indígenas a compartir saberes, experiencias, rituales y tradiciones en el Festival
Ipanoré; éste es un evento que protege y difunde el patrimonio cultural de los habitantes de la región y en el que se aprecia la diversidad etnográfica del Vaupés (Caicedo y Barreto, 2016).

Actualmente hay planes por parte de la gobernación con el fin de reconstruirla, pues este era un atractivo de visita obligatoria para los visitantes, un punto de referencia para la exposición de las manifestaciones culturales de la región.

El malecón, aunque reciente en su construcción, ha sido usado como sitio para el consumo de sustancias psicoactivas y alcohol además de baño público por algunas personas haciendo que deje de ser una experiencia agradable para propios y foráneos.

Diversas comunidades han abandonado sus áreas para desplazarse a la ciudad, por lo cual algunos caminos se encuentran en mal estado y algunos caños son afectados con basuras de los visitantes; quienes disfrutan de estos caños los fines de semana hacen comidas y usan recipientes desechables sin tener en algunos casos el más mínimo cuidado recogerlos y tirarlos en los depósitos de basura.

Lo cual resulta en una inminente necesidad de poner en común acuerdo a organismos públicos y privados para de esta manera generar campañas de concientización en el cuidado del medio ambiente puesto que este descuido ha causado un impacto irreversible en la naturaleza, especialmente de los lugares más cercanos a los asentamientos urbanos.

\section{Aspectos Internos}

\section{Fortalezas}

\section{Diversidad Natural y Cultural}

Se ha puesto de manifiesto la diversidad étnica y cultural de este territorio lleno de monumentos naturales dignos de admiración. Confiada por parte de la ancestral anaconda que mientras arrumbaba por el río Vaupés y el gran raudal de jirijirimo se segmentaba fecundado esta tierra con los primeros gérmenes de vida, heredándoles en actos ancestrales de inmersión particulares lenguas, ritos y semblantes a cada una de las 27 familias que plantó; les dio conocimientos para mantener la armonía entre el hombre y la naturaleza, con la posibilidad de comunicarse con los espíritus que cuidan cada ser vivo y reorientar los flujos de energía. (Correa, 1983) 
Esta muestra de cosmovisión permite ver como se formaron nuevos asentamientos entre cerros y las laderas del rio Vaupés, entre la selva y sabanas, entre los grandes raudales y cachiveras; todo este patrimonio lo pueden disfrutar los turistas que se acercan a este territorio.

\section{Recurso diferenciador}

El departamento del Vaupés gracias a sus características puede realizar una oferta diversa en cuanto a productos turísticos tales como: el turismo de aventura, ecoturismo, turismo étnico, cultural y gastronómico, actividades como el senderismo, avistamiento de aves, canotaje por los rápidos del rio Vaupés, camping, hospedarse en una comunidad donde le den muestras al turista de sus expresiones culturales, sus danzas y rituales mientras prueba el casabe, la fariña, quiñapira y chicha y así ver un rojo amanecer amazónico en las playas del rio, donde pueden practicar futbol y voleibol playa para después desplazarse sobre el malecón hacia el centro de Mitú.

Determinando un producto versátil a un turista nacional o extranjero que busque experiencias diferenciadas.

\section{Nuevas inversiones en infraestructura}

En Mitú, la capital, los entes gubernamentales han hecho inversiones para el equipamiento de las zonas urbanas de infraestructura para el turismo, deporte y la cultura permitiendo una propicia recepción de todo tipo de visitantes.

\section{Debilidades}

\section{Escasa existencia de producto turístico desarrollado}

Las entidades gubernamentales del Departamento y las comunidades como entes autónomos no han desarrollado productos turísticos, después de un proceso de evaluación objetivo de los lugares y expresiones culturales aprovechables; la excepción es la comunidad Ceima Cachivera, ya que unos docentes del SENA crearon paquetes turísticos que ofertan el acceso a la Cachivera "Con apoyo del SENA la comunidad ha trabajado en la adecuación de senderos, manejo de basuras, embellecimiento del espacio público, control de ganado, establecimiento de bancos de semillas" (SENA, 2012), lo mismo que caminatas ecológicas al cerro flechas y por un valor adicional muestras culturales y gastronómicas. Sin embargo, aún no puede ser considerada una oferta integral, pues no proporciona opciones de transporte para distancia locales y nacionales.

\section{Insuficiente penetración en los canales de comercialización} turística

Los canales de comercialización son los medios por los cuales es posible ofertar, ya sea de forma masiva o individual, productos y servicios a un segmento específico de personas; pueden ser directos o indirectos (Damián, 2009).

El medio directo más común y eficaz es internet que podría ser aprovechado por los representantes de las comunidades y gobierno a través de páginas web, blogs, redes sociales, paginas oficiales (Gubernamentales) y publicidad pagada así como ofertar de manera rápida cualquier producto, servicio, actividad o lugar, reduciendo costos y trabajo evitando la intermediación de otros agentes.

\section{Infraestructura para el transporte $y$ altos costos}

Una de las debilidades de esta región es la escasa infraestructura para el transporte.El Vaupés no cuenta con vías terrestres interdepartamentales lo que provoca que mientras otras zonas de Colombia se desarrollan política, social y económicamente. (Zaninovich, 2017)

Adicionalmente su característica selvática su gran extensión y protección del territorio bajo la figura de resguardo indígena (Artículo 21, decreto 2164 de 1995), dificulta la comunicación con los demás municipios y corregimientos del departamento, haciendo más complejo el desplazamiento de locales y turistas por estas tierras.

Se cuenta con una terminal aérea por donde se introducen los bienes y servicios para el comercio además por donde entran y salen las personas desde y hacia el interior del país. Sin embargo los precios de los vuelos son elevados especialmente para los turistas o personas no nacidas en el territorio ya que puede llegar a pagar un vuelo desde Bogotá o Villavicencio más costoso que un vuelo a Panamá o Ecuador, lo que incrementa los costos y entorpece el desarrollo del turismo.

\section{Falta de apoyo gubernamental}

Durante el trabajo de observación realizado y la visita a las comunidades los indígenas relataban la falta de atención de la alcaldía y gobernación en cuanto a sus necesidades justificándose en un escaso presupuesto ya asignado. Por demás se nota el desinterés y descon- 
fianza por los proyectos de las comunidades, las cuales podrán alcanzar de estas entidades asesoría pero no inyección de capital.

\section{Organización de las comunidades}

Las comunidades son apáticas a organizarse homogéneamente para el desarrollo de los proyectos y actividades, cada una cuenta con un capitán, quien es su representante y que debe aprobar desde el ingreso a la comunidad hasta la actividad más transformadora; algunas veces aunque el capitán este de acuerdo con alguna situación u oferta los demás pueden mostrar apatía dificultando cualquier proceso.

Así mismo la impaciencia de algunos indígenas es una de los factores a resolver ya que esperan resultados de manera inmediata sin dar paso a los procesos burocráticos establecidos; la corrección es posible mediante un acompañamiento y capacitación donde se le dé una idea clara del progreso paulatino que debe tener este tipo de proyectos.

\section{Estrategias}

Para la solución y potenciación de los anteriores postulados, apoyados por el manual de desarrollo del ecoturismo creado por "The Nature Conservancy", se realizó la formulación del "Plan para el Manejo del Ecoturismo en el Municipio de Mitú y las Comunidades Indígenas Aledañas", que busca estructurar el sector turístico del departamento con 6 grandes estrategias y algunas otras propuestas que aportan puntos claves para el desarrollo del mismo (APTAE, 2002).

\section{Primer Estrategia}

Se definió la zonificación de uso para los visitantes; su objetivo es reseñar los lugares de uso intensivo, extensivo y la zona primitiva. La zona de uso intensivo es aquella donde por el bajo impacto ambiental puede concurrir una gran cantidad de personas en el mismo momento, a su vez en la zona de uso extensivo por el alto impacto ambiental se concentra un número determinado de visitantes; y en la zona primitiva la concentración de biodiversidad y protección legal no permite la actividad turística para preservarla (Fundación Senda Darwin, 2004).

En este mapa se pueden ubicar cada uno de los atractivos turísticos de las comunidades en la que se concentra este estudio, a los cuales se les ha asignado letras minúsculas con el fin de ser relacionados con los nombres consignado en la parte inferior. De la misma manera las comunidades indígenas están enumerados del 1 al 8 (estos números no tienen relación con su cercanía ni importancia), los cuales se pueden relacionar en la parte inferior donde encuentra el nombre de las comunidades. Se encuentra también la división político administrativa tradicional, es decir, las asociaciones a las cuales pertenecen dichas comunidades las cuales se les han relacionado con letras mayúsculas encerradas por en círculos de color verde. Se puede también apreciar los caños y ríos que bañan las tierras

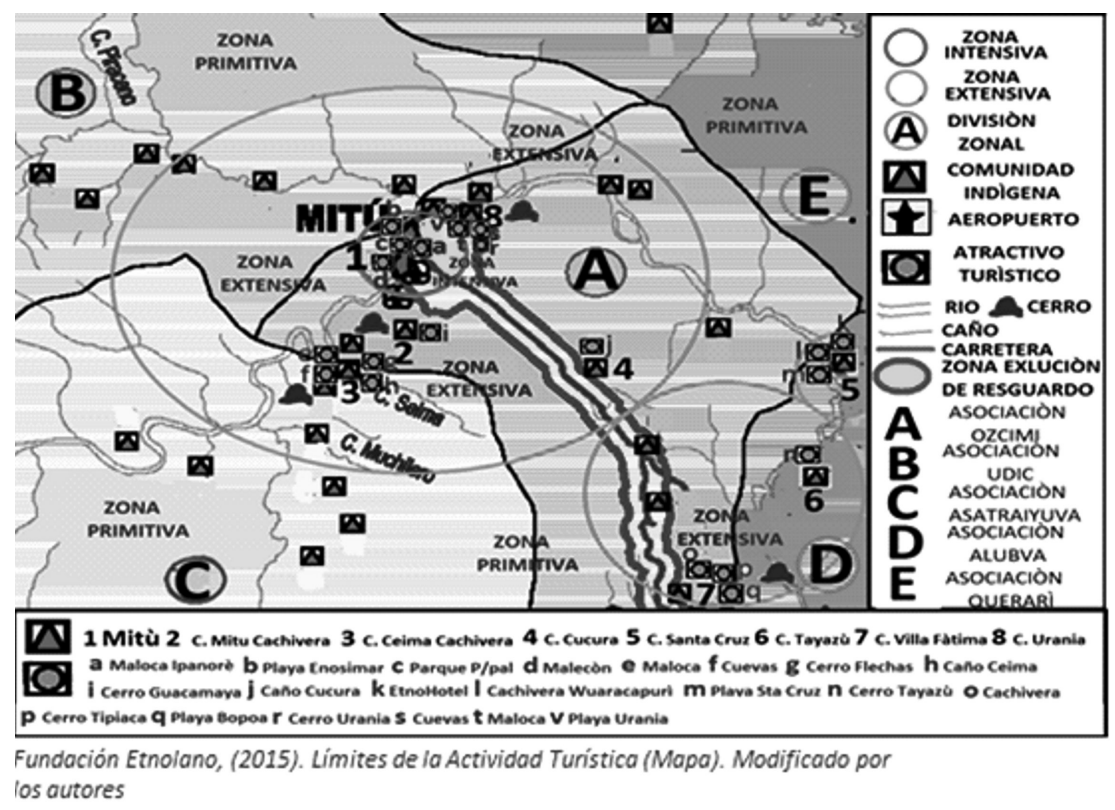

Figura 1. Zonificación del municipio de Mitú. 
vaupenses y los cerros que hacen parte de los atractivos valorados.

Por último y muy importante, se ha señalado las zonas con actividad intensiva, extensiva y primitiva. Las zonas de uso intensivo son las que concentran el uso público de alto impacto, en general, son muy pocas en el área, y representan menos del uno por ciento del territorio (The Nature Conservancy, 2004).

La zona intensiva se encuentra encerrada por un círculo rojo y es el área urbana de Mitú, donde hay mayor concentración de actividad tanto de locales como visitantes tales como: el malecón, las playas del rio Vaupés, el parque principal y anteriormente la maloca Ipanoré. En ellos se desarrollan actividades culturales y deportivas de gran importancia y que pueden ser incluidas en productos a ofertar en el mercado turístico; una de las causas por las cuales el uso de estas zonas es intenso es el fácil acceso, pues no existe ningún tipo de restricción para el ingreso y el uso de estos.

Las zonas extensivas o moderadas son las orientadas hacia el bajo impacto y en la cual se encuentran los senderos para visitantes en general; son más grandes que las anteriores pero sin embargo, representan sólo una parte menor del territorio (The Nature Conservancy, 2004). Es decir, esta zona comprende las áreas rurales cercanas a la capital del departamento, donde se concentran las comunidades con atractivos potenciales como lo son los cerros, cachiveras, playas y caños en cuanto a la parte natural y las malocas, bailes, gastronomía y lenguas en lo cultural; son áreas donde se mezcla la selva, con las sabanas y el contexto rural con caminos rudimentarios y de poco tránsito. Estos lugares son menos concurridos principalmente por la distancia y el difícil acceso, en algunos hay restricciones para el ingreso pues se procura la conservación y por la falta de personal disponible para brindar información y guianza, se pueden distinguir en el mapa porque están encerradas por un círculo de color naranja.

Las zonas primitivas o intocables son aquellas en las que el uso público es muy bajo o inexistente; esto debido a su lejanía, por la fragilidad de sus recursos o inexistencia de actividades y atractivos (The Nature Conservancy, 2004). Estas se pueden distinguir en el mapa pues se encuentran fuera de los círculos que señalan las zonas anteriores; se localizan muy lejos de las áreas urbanas, son zonas selváticas en su mayoría, en donde no hay caminos transitables ni afluentes navegables que permitan un acceso conveniente y la comunicación constante con los territorios aledaños. Cada una de estas zonas debe tener un trato específico tanto para su aprovechamiento como para su conservación.

Actualmente no existe un mapa de entes oficiales con la información presentada con el cual se puedan hacer comparaciones y la necesaria retroalimentación para una distribución concertada de las zonas y que dé un panorama más amplio al turista de los lugares que podrá disfrutar y bajo que condicionamiento debe hacerlo.

\section{Segunda Estrategia}

Consiste en la planificación y diseño de los sitios de visita, en el cual se delimita las zonas en las cuales



Figura 2. Sitios turísticos. 
se concentrarán las actividades turísticas y en el que se reduce el foco geográfico de investigación, ya que se han seleccionado las comunidades indígenas en las cuales hay atractivos turísticos y que se encuentran cerca al municipio de Mitú, pues facilita la planeación y la ejecución del plan turístico. Se integran en él las comunidades de Santa Cruz, Villa Fátima, Mitú Cachivera, Ceima Cachivera, Mituseño Urania, Cucura y Santa Cruz. Por medio de una línea roja se registran los límites de la actividad turística.

En este paso también se determinan cinco acciones iniciales para la mejora y desarrollo de la infraestructura y el acondicionamiento turístico intermunicipal, desarrollo de la infraestructura vial intercomunitaria, el acondicionamiento de servicios públicos en estos territorios indígenas, la construcción de alojamientos con métodos de construcción tradicional dentro de las comunidades y la señalización de los atractivos urbanos y rurales de la zona de uso.

\section{Tercer estrategia}

Requiere del diseño de la arquitectura sustentable para la práctica del ecoturismo en el departamento, esta debe respetar algunos principios básicos como lo son: el material debe ser extraído de la selva de manera responsable y garantizando que sean recursos renovables, en cantidades precisas y en lugares donde abunde dicho material, todo lo que se use para la construcción deben ser con materiales que no afecten la naturaleza, se debe eliminar el concepto de desperdicio y tener claro el ciclo de uso de los materiales para reemplazar de manera oportuna y responsable en el momento que se requiera. El diseño responsable balancea las necesidades humanas con la capacidad del ambiente natural y cultural, este debe poder apro- vechar al máximo la energía solar, los recursos hídricos, las corrientes de aire, para eliminar al máximo el uso de energía artificial.

Se realizó para este caso un diseño en 3D como propuesta, creado con un software llamado sketchup, éste permite observar el alojamiento desde todos los ángulos, se puede ingresar dentro del mismo y ver su equipamiento. En este caso el alojamiento tiene puertas hechas de malla natural para que permita el paso de luz y aire y evita el ingreso de animales e insectos, tiene rejillas cubiertas también con malla natural para que mantenga fresco y el techo está elaborado de palma de caraná, que puesta de la manera correcta no permite la filtración de agua. Es recomendable también que el equipamiento sea autóctono para que la experiencia del turista sea lo más cercano al estilo de vida indígena, es decir, que se duerma en hamaca, en medio de un ambiente natural y que pueda degustar a su vez de la gastronomía local.

\section{Cuarta estrategia}

Se creó un mecanismo para la generación de ingresos que llegará a las comunidades indígenas, los entes gubernamentales y que se destinará para la conservación y mitigación del impacto generado del uso de los atractivos turísticos. Se definieron una serie de aranceles que se ajustan al contexto turístico departamental y se relacionan en la Tabla 6.

Actualmente, la única comunidad en el departamento que genera algunos ingresos por arancel de ingreso es la comunidad Ceima Cachivera, cada vez que alguien accede a su territorio para el uso de los atractivos debe realizar un pago de $\$ 15.000$ pesos colombianos por persona.

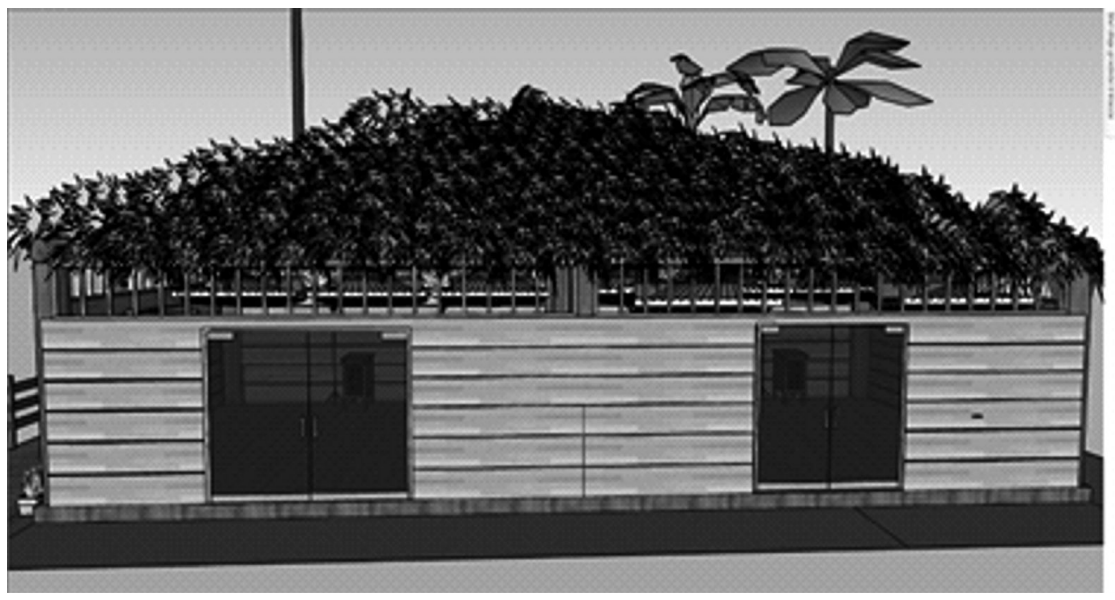

Figura 3. Diseño 3D de alojamiento indígena. 
Tabla 6. Mecanismo para la Generación de Ingresos, Lista de Aranceles.

\begin{tabular}{|l|l|}
\hline \multicolumn{1}{|c|}{ Tipo de cuota } & \multicolumn{1}{c|}{ Descripciòn } \\
\hline Aranceles de ingreso & $\begin{array}{l}\text { Como condición para el acceso a los atractivos } \\
\text { turísticos y las comunidades indígenas. }\end{array}$ \\
\hline Aranceles de admisión & $\begin{array}{l}\text { Pago por el uso de una instalación o por una actividad especial } \\
\text { tal como el avistamiento de aves o caminatas ecológicas. }\end{array}$ \\
\hline Aranceles de uso & $\begin{array}{l}\text { Valor que paga el visitante para usar las instalaciones tales } \\
\text { como estacionamientos, zonas de camping, centros de } \\
\text { información, uso de botes, uso de refugios, etc. }\end{array}$ \\
\hline Licencias y permisos & $\begin{array}{l}\text { Obligación para que firmas privadas de turismo operen en las áreas protegidas, } \\
\text { licencias para operadores turísticos, transportistas, guías, entre otros. }\end{array}$ \\
\hline Ganancias por ventas & Dinero de la venta de souvenires \\
\hline Aranceles de concesión & $\begin{array}{l}\text { Comisiones o parte de los ingresos recaudados por los concesionarios } \\
\text { que proporcionan servicios para los turistas en las áreas protegidas } \\
\text { como transporte, alquileres, tiendas de recuerdos. }\end{array}$ \\
\hline Impuestos & Para las habitaciones de los hoteles, el uso de los aeropuertos y vehículos. \\
\hline Ar. de arrendamiento y alquiler & $\begin{array}{l}\text { Cuotas por alquilar o arrendar propiedades o equipamiento } \\
\text { de las comunidades o propiedad del estado. }\end{array}$ \\
\hline Donaciones Voluntarias & $\begin{array}{l}\text { Incluyen dinero en efectivo, regalos "en especies" y trabajo, con frecuencia } \\
\text { se reciben a través de grupos del tipo "amigos de la naturaleza". }\end{array}$ \\
\hline
\end{tabular}

Elaborada por el autor como resultado del análisis del estado del turismo en Vaupés, Colombia (2017).

\section{Quinta Estrategia}

Hace relación al manejo y monitoreo del impacto de los visitantes, cada vez que uno de ellos pone un pie en el sitio turístico, causa un impacto negativo y este es un hecho inevitable, se crearon una serie de indicadores que permitan medir el impacto que genera el movimiento de los visitantes desde el punto de vista ambiental, experiencial, económicos y socio-culturales; la forma de medición es por cantidad máxima de veces que puede ocurrir en suceso en un determinado territorio, de manera que pueda medirse y esta medición genere una alerta en quienes administran el monitoreo. Se mostrarán a continuación solo unos ejemplos de ellos (Tabla 7):

Estos indicadores más los que se vayan creando a medida que se desarrollan las actividades y van apareciendo nuevas situaciones, permitirán la intervención oportuna en cada circunstancia para procurar el mayor beneficio para los individuos, las comunidades, poblaciones, entes gubernamentales y visitantes

\section{Sexta Estrategia}

Guías naturalistas; los guías naturalistas desempeñan un papel central en la implementación del concepto de ecoturismo, son ellos los que principalmente proporcionan el elemento educativo a la actividad ecoturística; son su capacidad y su compromiso los que aseguran que los impactos negativos del turismo sean minimizados pues tienen labores de conservación de recursos naturales y culturales.

\section{Discusión}

Se debe realizar una labor con el departamento y la alcaldía de legalización de las zonas turísticas según las características del territorio para su uso.

La participación de las comunidades tradicionales cercanas al área urbana de Mitú generará empoderamiento por parte de las misma y un atractivo adicional para los paquetes turísticos; de esta manera se harán participes de la oferta turística y para ello es necesario brindar apoyo en adecuación en unidades sanitarias, la construcción o mejoramiento de infraestructura habitacional según las costumbres ancestrales.

Es necesario capacitar a los indígenas en cuanto a la obtención de resultados del plan turístico; ya que éste es un proceso que requiere tiempos y movimientos para lograr las metas propuestas en el mediano y largo 
Tabla 7. Indicadores para el Manejo y Monitoria del Impacto Medio Ambiental de la Actividad Turística.

\begin{tabular}{|c|c|c|}
\hline Indicador & Información & $N^{\circ}$ de sucesos \\
\hline \multicolumn{3}{|c|}{$\begin{array}{ll}\text { Ambientales } \\
\end{array}$} \\
\hline $\begin{array}{l}\text { Erosión del Suelo en } \\
\text { un sitio en particular }\end{array}$ & $\begin{array}{l}\text { A cada comunidad le corresponde } \\
\text { determinar las medidas de su territorio en } \\
\text { kilómetros y a su vez el área de uso del } \\
\text { turismo con el fin de poder determinar } \\
\text { el porcentaje de erosión del suelo. }\end{array}$ & $\begin{array}{l}\text { El máximo aceptable de terreno con erosión } \\
\text { es del } 25 \% \text {, es decir que cada } 6 \text { o } 12 \text { meses } \\
\text { debe realizarse una evaluación y determinar si } \\
\text { la erosión ha sido casi nula (por ejemplo: }-5 \% \text { ), } \\
\text { media }(10 \%) \text { o se encuentra cerca del máximo. }\end{array}$ \\
\hline Fogatas Ilegales & $\begin{array}{l}\text { De llegar a este número antes del } \\
\text { año se deben implementar acciones } \\
\text { como mayor señalización en el área e } \\
\text { instrucciones más precisas a los visitantes. }\end{array}$ & $\begin{array}{l}\text { El máximo de eventos para este indicador es } \\
\text { de } 3 \text { en el área de uso para turismo al año. }\end{array}$ \\
\hline \multicolumn{3}{|c|}{$\begin{array}{ll}\text { Experienciales } \\
\end{array}$} \\
\hline $\begin{array}{l}\text { Cantidad de } \\
\text { encuentros }\end{array}$ & $\begin{array}{l}\text { El encuentro entre dos grupos de turistas } \\
\text { en actividades como avistamiento afecta } \\
\text { la experiencia del turista, debe tener } \\
\text { cada actividad un tiempo establecido }\end{array}$ & $\begin{array}{l}\text { El avistamiento de aves puede durar de } 3 \text { a } 4 \\
\text { horas, por tanto, pueden hacerse máximo } 2 \\
\text { avistamientos cada día, uno en cada jornada, } \\
\text { para evitar encuentro entre grupos. El máximo de } \\
\text { incidentes para este indicador es de } 2 \text { por mes. }\end{array}$ \\
\hline $\begin{array}{l}\text { Quejas sobre } \\
\text { visitantes ruidos }\end{array}$ & $\begin{array}{l}\text { En algunos atractivos ubicados en } \\
\text { sitios públicos, en las comunidades } \\
\text { y sus alrededores no está permitido } \\
\text { ningún tipo de ruidos extraños a los } \\
\text { generados por la actividad turística }\end{array}$ & $\begin{array}{l}\text { Para este indicador se permitirá un } \\
\text { máximo de } 2 \text { incidentes por mes. }\end{array}$ \\
\hline \multicolumn{3}{|c|}{$\begin{array}{ll}\text { Económicos } \\
\end{array}$} \\
\hline $\begin{array}{l}\text { Cantidad de } \\
\text { empresarios } \\
\text { ecoturísticos. }\end{array}$ & $\begin{array}{l}\text { Para evitar en algún momento la saturación } \\
\text { de operadores turísticos en el área, las } \\
\text { comunidades deben hacer las veces de } \\
\text { reguladores estableciendo convenios con } \\
\text { una cantidad determinada de intermediarios. }\end{array}$ & $\begin{array}{l}\text { Para este indicador el máximo es de } 2 \\
\text { intermediarios nuevos por año, de esta manera } \\
\text { es posible regular el número de ingreso, la } \\
\text { carga operativa y por tanto el impacto. }\end{array}$ \\
\hline $\begin{array}{l}\text { Ingresos por arancel } \\
\text { de entrada }\end{array}$ & $\begin{array}{l}\text { De no darse los resultados esperados } \\
\text { debe evaluarse las acciones } \\
\text { implementadas en la promoción de las } \\
\text { actividades turísticas en la región. }\end{array}$ & $\begin{array}{l}\text { Con la promoción que se debe hacer de los } \\
\text { lugares y atractivos turísticos los ingresos } \\
\text { deben incrementar por lo menos de un } 5 \text { a } \\
8 \% \text { año por año, por tanto, este indicador } \\
\text { debe evaluarse al término de cada año. }\end{array}$ \\
\hline \multicolumn{3}{|c|}{$\begin{array}{l}\text { Socio-culturales } \\
\end{array}$} \\
\hline $\begin{array}{l}\text { Subsistencia de } \\
\text { prácticas tradicionales }\end{array}$ & $\begin{array}{l}\text { La idiosincrasia indígena no debe } \\
\text { adaptarse a las costumbres occidentales, } \\
\text { sino que debe mantenerse y fortificarse } \\
\text { de tal modo que lo que perciba el turista } \\
\text { no sea un remedo de las prácticas } \\
\text { tradicionales sino una autentica muestra } \\
\text { de experiencias comunitarias ancestrales. }\end{array}$ & $\begin{array}{l}\text { De manera fraternal se debe hacer una revisión } \\
\text { del estilo de vida tratando de volver siempre a } \\
\text { las raíces y proponer un plan de fortalecimiento } \\
\text { cultural especialmente para las nuevas } \\
\text { generaciones. Por lo menos una vez al año. }\end{array}$ \\
\hline $\begin{array}{l}\text { Cantidad de } \\
\text { violaciones a la } \\
\text { seguridad }\end{array}$ & $\begin{array}{l}\text { Para el ingreso a algunos atractivos en el } \\
\text { departamento, en los que se requiere de } \\
\text { un permiso de parte de los capitanes y en } \\
\text { algunas ocasiones del pago de un arancel; } \\
\text { en su mayoría no cuentan con un cerco } \\
\text { que evite el ingreso ilegítimo de personas. }\end{array}$ & $\begin{array}{l}\text { El máximo aceptado es de } 3 \text { sucesos por mes, } \\
\text { los regentes de los lugares deben apoyarse } \\
\text { de las entidades de fuerza pública para el } \\
\text { control y correctivo en estas situaciones. }\end{array}$ \\
\hline
\end{tabular}

Elaborada por el autor como resultado del análisis del estado del turismo en Vaupés, Colombia (2017). 
plazo; las comunidades por su cosmovisión pretenden obtener resultados en el corto plazo pero un desarrollo de este tipo requiere de etapas más amplias.

Se propone combinar los diseños indígenas con la modelación computarizada para obtener y presentar estructuras habitacionales confortables y llamativas pero conservando todos los elementos ancestrales que permiten una experiencia más cercana al contexto vaupense.

Se deberá reglamentar una serie de tarifas y tasas para el uso de los servicios ecosistémicos; estos deben de desarrollarse por parte de los entes gubernamentales en el marco del plan de turismo del Vaupés, permitiendo general recursos tanto para el mantenimiento como para el sustento de las comunidades participantes.

Es necesario establecer convenios de formación con entidades como el SENA para apoyar los procesos de guiansa en las comunidades indígenas; ya que estas poseen los conocimientos ancestrales de preservación pero que deben ser aterrizados para los turistas foráneos.

El cuello de botella para el desarrollo turístico es la forma de acceso hacia Mitú, ya que la única vía es aérea; se hace necesario e imprescindible la participación por parte del gobierno central con el mejoramiento de las rutas aéreas a través de la aerolínea estatal (Ley 1427 de 2010) en pro del cumplimiento del plan turístico propuesto.

Dentro del desarrollo del plan es necesario implementar formación a nivel técnico y profesional en cuanto a las nuevas alternativas de turismo sostenible.

\section{Agradecimientos}

A María Mercedes Trinidad Carrero quien desarrolló el trabajo de campo y abrió las puertas hacia las comunidades indígenas.

\section{Referencias}

AIEST (1991). 41 Congreso de International Association of Scientific Experts in Tourism. AIEST. Alemania.

APTAE. (2002). Asociacion Peruana de Turismo de Aventura, Ecoturismo y Turismo Especializado. Obtenido de Manual para el Desarrollo del Turismo: http://www.aptae.pe/archivos_up/0100-introduccion-a-la-planificacion-del-ecoturismoandy-drumm-y-alan-moore.pdf

Artículo 21, decreto 2164 de 1995, Por el cual se reglamenta parcialmente el Capítulo XIV de la Ley 160 de 1994 en lo relacionado con la dotación y titulación de tierras a las comunidades indígenas para la constitución, reestructuración, ampliación y saneamiento de los Resguardos Indígenas en el territorio nacional.

Bertoni M. Turismo sostenible: su interpretación y alcance operativo. Cuad Geogr Rev Colomb Geogr. 2008;(17):155-163.

Cabrera-Becerra G. Los trabajos etnográficos de Peter van Emst, Mario y Michel Terribilini en el Noroeste amazónico en la segunda mitad del siglo XX. Boletín de Antropología Universidad de Antioquia. 2014;29(47):96-115.

Caicedo JM, Barreto Castañeda LA. 2016. plan de desarrollo departamental. obtenido de gobernaciòn del vaupès: www.gobernaciondelvaupes.com

Chávez Dagostino R, Andrade Romo E, Espinoza Sánchez R. Turismo y desarrollo sustentable: contribución de Hispanoamérica. Teoría y Praxis. 2013;(13):9-33.

Colombia Aprende. (Marzo de 2016). PLAN DE ATENCION INTEGRAL A LA PRIMERA INFANCIA. Obtenido de http://www. colombiaaprende.edu.co/html/familia/1597/articles-305955_ vaupes.pdf

Colombia.co. (2016). Colombia.co. Obtenido de Festival Ipanorè: http://www.colombia.travel/es/ferias-y-fiestas/festival-ipanore

Correa F. Elementos de identidad y organización social entre las comunidades indígenas de la región del Vaupés. Maguaré. 1983;2:97-123.

Damián D. (Diciembre de 2009). Comercializacion y Gestión de Ventas. Obtenido de Canales de Distribuciòn: http://comercializacionyventas-apuntes.blogspot.com.co/2009/12/canales-dedistribucion.html

Departamento Nacional de Planeación. (2015). Información General del Departamento. Obtenido de https://colaboracion. dnp.gov.co/CDT/Inversiones\%20y\%20finanzas\%20pblicas/ Vaup\%C3\%A9s\%2015.pdf

Echandía Castilla C. La Guerra por el Control Estratégico en el Suroccidente Colombiano. Revista Sociedad y Economía. 2004;(7):65-89.

Fundación Senda Darwin. (2004). Desarrollo del Ecoturismo. Obtenido de Volumen II: http://www.sendadarwin.cl/espanol/ wp-content/uploads/2010/04/manual-desarollo-del-ecoturismo-vol-2.pdf

Gallego Acevedo, L. ¿Cultura para consumir? Los yagua y el turismo cultural en el Trapecio Amazónico. Revista Colombiana de Antropología. 2011;47(1):113-136.

Gobernación del Vaupés. (22 de Julio de 2017). Información general del departamento. Obtenido de http://www.vaupes.gov.co/departamento/nuestro-departamento

Hernandez Sampieri R. (2010). Capitulo IV Metodología. McGrawHill.

Jiménez DA. Construyendo agenda 21 para el departamento de Vaupés: una construcción colectiva para el desarrollo sostenible de la Amazonia Colombiana.

Instituto Amazónico de Investigaciones Científicas" SINCHI".2007. 
Ley 1427 de 2010, por medio de la cual se modifica la naturaleza juridica de la empresa servicios aereos a territorios nacionalesSATENA.

López-Bonilla JM, López-Bonilla LM. La capacidad de carga turística: Revisión crítica de un instrumento de medida de sostenibilidad. El Periplo Sustentable. 2008;15:123-150.

Luck M, Kirstages T. (eds) (2002): Global ecotourism policies and case studies perspectives and constraints. Channel View Publications. Clevedon, 216 pp.

Moya MB. Turismo vivencial: un ejemplo responsable sin ingredientes artificiales. Investigación y análisis. 2006.

Narváez F, Blanco-Sepúlveda R. El ecoturismo como estrategia de desarrollo rural en América Latina. Caso de la aldea indígena plan grande quehueche (izabal, guatemala). Espacio, Tiempo y Forma. Serie VI, Geografía. 2002;15:109-127

Nello Andreu MG. (2003): Ecoturismo, conservación de la naturaleza y desarrollo local. El caso de México, América Central y las Grandes Antillas. Tesis Doctoral. Universitat Rovira i Virgili.

Organización Mundial del Turismo (1998). Guía para administradores locales: desarrollo turístico sostenible. OMT. España 1998.
Ponce Talancón, H.La matriz foda: alternativa de diagnóstico y determinación de estrategias de intervención en diversas organizaciones. Enseñanza e Investigación en Psicología. 2007;12(1):113-130.

SENA, Servicio Nacional de Aprendizaje. Etnoecoturismo: Encanto de vida natural y cultural en Ceima Cachivera Comunidad de Ceima Cavhivera, Vaupés. 2012

Tarlombani da Silveira M. Turismo y sustentabilidad: Entre el discurso y la acción. Estudios y Perspectivas en Turismo. 2005;14(3):222-238.

Trejo Castro J, Marcano Navas N. Ecoturismo y Geoturismo: alternativas estratégicas para la promoción del turismo ambiental sustentable venezolano. Revista de Investigación. 2016;40(88):202-228.

The Nature Conservancy. (2017). Mundo TNC. Obtenido de http://www.mundotnc.org/sobre/quienes-somos/index. htm?intc=mundo.tnav.sobre

Zaninovich D. Vías regionales y la necesidad de una gestión compartida. Revista de Ingeniería. 2017;(45):72-79. 